Revista Colombiana de Obstetricia y Ginecología Vol. 55 No.4 • 2004 • (315-320)

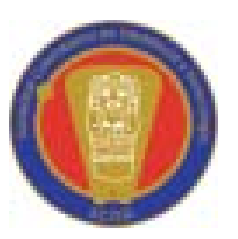

SERIE DE CASOS

\title{
LAPAROSCOPIA GINECOLÓGICA EN ADOLESCENTES EN TRES INSTITUCIONES DE CALI, 1998-2003
}

\section{GYNECOLOGIC LAPAROSCOPY PERFORMED ON ADOLESCENTS AT \\ THREE INSTITUTIONS IN THE CITY OF CALI, COLOMBIA. 1998-2003}

Alba Lucía Mondragón, M.D. * Gloria Amparo Viáfara, M.D.**,

Beatriz Vinueza, M.D.***

Recibido: julio 7/04 - Revisado: septiembre 13/04 - Aceptado: octubre 26/04

\section{RESUMEN}

Introducción: el desconocimiento de la caracterización de cirugía laparoscópica ginecológica en adolescentes en nuestra ciudad, abre la necesidad de su investigación para el posterior planteamiento de sugerencias y estrategias de implementación de esta tecnología quirúrgica.

Materiales y métodos: se revisaron las historias clínicas de 37 mujeres adolescentes menores de 20 años quines fueron sometidas a cirugía laparoscópica en tres importantes centros de la ciudad de Cali entre 1998 - 2003. Los registros fueron analizados en el programa Estadístico Epi Info 6.

Resultados: de 3.448 procedimientos 37 $(1,1 \%$ fueron hechos en adolescentes. La edad promedio fue 16 años. La indicación más frecuente fue el dolor pélvico $(54,1 \%)$, seguido por dismenorrea e infertilidad en un $10,8 \%$ cada una y masa anexial en un $8,1 \%$. Los hallazgos quirúrgicos más frecuentes fueron endometriosis en 11 adolescentes $(29,7 \%)$ siguiendo en frecuencia: ovarios anovulatorios $(16,2 \%)$ y quistes anexiales $(13,5)$. Llamó la atención encontrar

Trabajo presentado y aceptado en el XIV Congreso Nacional de Obstetricia y Ginecología en la modalidad de poster.

* Medicina y Cirugía, Universidad del Valle, Especialista en Ginecología y Obstetricia, Universidad del Valle. Especialista en Medicina de la Reproducción, Universidad de Ginebra, Suiza. Ginecóloga programa de infertilidad, Profamilia. Ginecóloga, Seguro Social. Correo electrónico: albamon@hotmail.com.

** Medicina y Cirugía, Universidad del Valle, Especialista en Ginecología y Obstetricia, Universidad Pontificia Bolivariana (Medellín). Ginecóloga Susalud.

***Residente de Ginecología y Obstetricia, Universidad Libre, Cali. complicaciones de utilización de DIU, embarazo ectópico, graves secuelas de EPI y varicocele.

Conclusiones: es importante promover la utilización de esta técnica quirúrgica, minimamente invasiva, en la población adolescente. Se recomienda estandarizar el registro de recolección de datos de las historias clínicas prequirúrgicas, intraoperatorias y posquirúrgicas para futuros estudios multicéntricos prospectivos.

Palabras claves: laparoscopia, salud de los adolescentes, endometriosis, dolor pélvico.

\section{SUMMARY}

Introduction: as no data had been collected regarding the characteristics of gynaecologic laparoscopic surgery performed on adolescents in Cali, this led to research concerning this surgical technology, followed by suggestions being made for improving it and strategies proposed for implementing its use.

Materials and methods: the clinical histories of 37 adolescent females ( $<20$ years old) who underwent laparoscopic surgery in 3 clinics in Cali between 1998 and 2003 were studied. The data was analysed by using Epi Info 6.0 software.

Results: 3,448 gynaecological laparoscopic surgeries were carried out during the period being studied; 37 (1.1\%) patients were adolescents. This group's mean age was 16 . The reasons given for performing laparoscopic surgery were: pelvic pain (54.1\%), dysmenorrhea (10.8\%), infertility (10.8\%) and pelvic mass (8.1\%). Laparoscopic findings were: 
endometriosis (29.7\%), anovulatory ovaries (16.2\%) and adnexal cysts (13.5\%). Unusual findings were: DIU complications, ectopic pregnancy, pelvic inflammatory disease and varicocele.

Conclusion: this type of surgery, having minimal invasive impact, should be promoted for use on adolescents. We suggest that all hospitals standardise their pre-surgery, intra-operation and post-surgery clinical histories to facilitate carrying out future multi-centre prospective research.

Key words: laparoscopy, laparoscopic surgery, adolescents, endometriosis, pelvic pain.

\section{INTRODUCCIÓN}

La cirugía laparoscópica es un procedimiento de mínima invasión, que permite una rápida recuperación, excelente resultado cosmético y menor dolor, características que la hacen muy atractiva para el manejo de diversas patologías. Su uso se ha generalizado a diversos procedimientos tanto en cirugía general como en ginecología donde es ampliamente utilizada en infertilidad, endometriosis, síndrome adherencial y dolor pélvico entre otras. ${ }^{1}$ Por sus ventajas innegables también ha penetrado en el campo de la cirugía infantil y del adolescente donde es informada con una frecuencia que oscila entre las diferentes series de 1,7 a 5\% de todas las cirugías laparoscópicas realizadas. ${ }^{1-5}$

No se ha descrito la frecuencia de realización de este procedimiento y sus indicaciones en la ciudad de Cali, por lo tanto el objetivo del presente trabajo es describir las características del uso de la laparoscopia en un grupo de mujeres menores de 20 años, para el posterior planteamiento de sugerencias y estrategias de implementación de esta tecnología quirúrgica.

\section{MATERIALES Y MÉTODOS}

Diseño: Serie de casos. Población: pacientes menores de 20 años a quienes se les practicó laparoscopia en 3 instituciones de salud (Profamilia, Clínica de los Andes y Clínica del Seguro Social) en la ciudad de Cali en el periodo comprendido entre 1 de enero de 1998 y 31 de marzo de 2003. Se excluyó del estudio la población infantil por encontrarse reporte solamente de un caso de laparoscopia realizada en el ISS a una niña de 5 años a la cual se practicó gonadectomía profiláctica por presentar síndrome de feminización testicular.

\section{Procedimiento}

Se revisaron los registros estadísticos de las pacientes, así como las historias clínicas y los registros sistematizados, previo a la autorización de cada una de las instituciones. Los datos fueron diligenciados en un cuestionario. La información recolectada fue codificada y llevada a una base de datos EXCEL, en doble captura para evitar un sesgo de información.

\section{Equipo}

El instrumental utilizado en las tres clínicas fue el mismo equipo empleado para la cirugía laparoscópica de la mujer adulta, canal operatorio de 10 $\mathrm{mm}$, la mayoría trócares metálicos, trócares laterales de $5 \mathrm{~mm}$ también metálicos y cánula intrauterina de Rubin. Sólo en una de las clínicas se tuvo acceso a la utilización de láser. No hay descripciones de presiones intrabdominales máximas utilizadas.

\section{Definición de términos}

\section{Laparoscopia operatoria}

Laparoscopia en la cual se realizó algún procedimiento intraabdominal guiado bajo visión con cámara y video, utilizando anestesia general.

\section{Laparoscopia directa}

Laparoscopia en la cual sólo se utilizó el laparoscopio bajo visión directa del cirujano, sin utilización de video y utilizando sólo sedación de la paciente y anestesia local en el sitio de la incisión infraumbilical.

\section{Laparoscopia video-diagnóstica}

Laparoscopia bajo visión con cámara y video, utilizando anestesia general, pero sin realización de ningún procedimiento operatorio intraabdominal o pélvico. 


\section{Desenlaces a medir}

Entidad institucional, edad, síntomas clínicos, hallazgos al examen físico, diagnóstico prequirúrgico, tipo de laparoscopia, hallazgos quirúrgicos, procedimientos, complicaciones y tiempo quirúrgico.

Análisis: para observar la consistencia y revisar los datos se realizó un análisis exploratorio doble. Se procedió luego al análisis aplicando el Software Epinfo 6, los datos se presentan en cuadros, se utilizan medidas de tendencia central y dispersión para variables continuas y porcentajes para las categóricas.

Limitaciones para la recolección de la información:

1. Las historias clínicas de las pacientes de una de las clínicas no reposan en el archivo por lo cual no pudieron ser revisadas en su totalidad.

2. Los datos de las pacientes de una de las instituciones fueron obtenidos de la base de datos sistematizados que allí reposa, al no tener acceso a las historias clínicas no se pudieron recoger todos los datos requeridos.

3. En una de las clínicas estos datos están codificados por grupos de edad. El primer grupo comprende mujeres de menos de 15 años, el siguiente grupo comprende mujeres entre 16 a 24 años. Se tomó solamente el grupo de menores de 15 dejando un subregistro de las jóvenes entre 16 y 19 años que estaban dentro del siguiente grupo pero que no hubo forma de poder identificar.

\section{RESULTADOS}

\section{Características generales}

De un total de 3.448 mujeres que fueron intervenidas quirúrgicamente vía laparoscópica por indicación ginecológica, se hallaron 37 mujeres adolescentes, que representan solamente el 1,1\% del total de cirugías laparoscópicas en menores de 20 años. La mayoría de las pacientes $(62,2 \%)$ provenían de la Clínica de Profamilia (tabla 1).

Excluyendo las jóvenes de una de las clínicas donde la edad es registrada como «menores de 15», se encontró que la edad promedio del resto de las adolescentes fue de 16,89 \pm 1,77; la mayoría de ellas tenían más de 15 años (este estimador sólo aplica al 32\% de la muestra).

Si se excluyen los datos de una de las clínicas en las cuales no se consignó información al respecto (43\%), encontramos que $75 \%$ de las adolescentes que fueron llevadas al procedimiento habían iniciado relaciones sexuales.

El 62,2\% fueron laparoscopias operatorias, el $32,4 \%$ fueron laparoscopias directas y el 5,4\% fueron video diagnósticas.

El promedio del tiempo en minutos empleado fue de 31,82 $\pm 22,11$, el tiempo máximo fue de 75 minutos, durante los cuales se realizaron procedimientos como cistectomía o salpinguectomía y los tiempos mínimos de 5 y 7 minutos que fueron empleados en laparoscopias diagnósticas.

No se pudo hacer una adecuado análisis de las técnicas quirúrgicas por el subregistro y la diferencia de recolección de los datos en las tres clínicas.

\section{Indicaciones, hallazgos y procedimientos}

Se encontró que 54,1\% de las adolescentes manifestaron la presencia de dolor pélvico y el $10,8 \%$ dismenorrea, lo que representa una indicación de laparoscopia por dolor de 64,9\%. A pesar de ser población adolescente, 4 presentaban infertilidad $(10,8 \%)$ de ellas la mitad era de tipo primaria y la otra mitad secundaria (tabla 2). En el

Tabla 1. Laparoscopia en adolescentes según institución en Cali 1998-2003.

\begin{tabular}{lcccc} 
Clínica & $\begin{array}{c}\text { No. total de } \\
\text { laparoscopias }\end{array}$ & $\begin{array}{c}\text { No. en } \\
\text { adolescentes }\end{array}$ & $\begin{array}{c}\text { \% en } \\
\text { adolescentes }\end{array}$ & $\begin{array}{c}\text { \% de aporte } \\
\text { a la clínica }\end{array}$ \\
\hline Profamilia & 757 & 23 & 3 & 62,2 \\
\hline Los Andes & 2.327 & 11 & 0,5 & 29,7 \\
\hline ISS & 364 & 3 & 0,82 & 8,1 \\
\hline Total & 3.448 & 37 & 1,1 & 100 \\
\hline
\end{tabular}


examen físico ginecológico, en el 29,7\% no se encontró ninguna anormalidad; en las restantes el hallazgo más frecuente fue dolor a la palpación de los anexos $(10,8 \%)$.

El hallazgo quirúrgico más frecuente fue endometriosis; de éstas 9 fueron clasificadas como leve (24,3\%), 1 moderada y 1 con endometrioma ovárico que corresponden al 2,7\% cada una. El resto de hallazgos se presentan en la tabla 3. No se encontró ninguna patología en $10,8 \%$ de las pacientes.

El procedimiento operatorio más frecuente durante la laparoscopia fue la fulguración de focos ó endometriósicos. Los otros procedimientos operatorios se observan en la tabla 4. En algunos

\section{Tabla 2. Indicaciones de laparoscopia} en adolescentes. 1998-2003

\begin{tabular}{lcc} 
Indicación & No. & $\%$ \\
\hline Dolor pélvico & 20 & 54,1 \\
\hline Dismenorrea & 4 & 10,8 \\
\hline Infertilidad & 4 & 10,8 \\
\hline Masa anexial & 3 & 8,1 \\
\hline Amenorrea primaria & 2 & 5,4 \\
\hline Embarazo ectópico & 1 & 2,7 \\
\hline Malformación congénita & 1 & 2,7 \\
\hline DIU en cavidad pélvica & 1 & 2,7 \\
\hline Sin dato & 1 & 2,7 \\
\hline
\end{tabular}

Tabla 3. Hallazgos quirúrgicos de laparoscopia en adolescentes. 1998-2003

\begin{tabular}{lcc} 
Hallazgos & No. & $\%$ \\
\hline Endometriosis & 11 & 29,7 \\
\hline Ovarios anovulatorios & 6 & 16,2 \\
\hline Quiste ovárico & 3 & 8,1 \\
\hline Malformación congénita & 3 & 8,1 \\
\hline Miomatosis & 3 & 8,1 \\
\hline Varicocele & 2 & 5,4 \\
\hline Quiste paratubárico & 2 & 5,4 \\
\hline Embarazo ectópico & 1 & 2,7 \\
\hline $\begin{array}{l}\text { Adherencias, hidrosálpinx, } \\
\text { obstrucción tubárica }\end{array}$ & 1 & 2,7 \\
\hline DIU en cavidad pélvica & 1 & 2,7 \\
\hline Ninguno & 4 & 10,8 \\
\hline
\end{tabular}

casos, la sección de útero sacros acompañó a otro procedimiento o se hicieron varios procedimientos en la misma paciente. En 35\% de los casos no se realizó ningún procedimiento quirúrgico laparoscópico.

En los casos en que se pudo acceder a las historias clínicas, se encontraron dos complicaciones: un hematoma de herida quirúrgica, el cual fue drenado sin complicaciones y un caso de dolor posquirúrgico severo. Debido a que sólo se pudo acceder al 22\% de las historias, esta información no permite comparar ni sacar conclusiones.

\section{DISCUSIÓN}

La primera información sobre técnicas laparoscópicas en niños se remonta a dos décadas atrás y son publicaciones anecdóticas. Gans y Berce en 1973 y algunas series de casos Cognat Rosenberg, David en 1973. ${ }^{6}$ Inicialmente Leaders Gans, Rodgers, Georgeson y Lobe mostraron que se podía aplicar la laparoscopia operatoria a niños utilizando los instrumentos de tamaño para adultos. En la medida que aumentó la demanda de cirugías en infantes la industria comenzó a fabricar instrumental más pequeño y corto para uso pediátrico. ${ }^{7}$ Posteriormente en 1980 se introdujo la cirugía de mínimo acceso a la cirugía general y los cirujanos pediatras han innovado las técnicas endoquirúrgicas para aplicarla a la cirugía infantil, generalizándose para

\section{Tabla 4. Procedimientos quirúrgicos de laparoscopia en adolescentes. 1998-2003}

\begin{tabular}{lcc}
\hline Procedimiento & No. & \%* \\
\hline Fulguración focos endometriosis & 11 & 29,7 \\
\hline Sección de útero sacros & 8 & 21,6 \\
\hline Cistectomía & 3 & 8,1 \\
\hline Salpiguectomía & 2 & 5,4 \\
\hline Varicocelectomía & 1 & 2,7 \\
\hline Termólisis mioma & 1 & 2,7 \\
\hline Drilling de ovario & 1 & 2,7 \\
\hline Drenaje y fulguración cápsula quiste & 1 & 2,7 \\
\hline Corte septo intrauterino & 1 & 2,7 \\
\hline Extracción de DIU abdominal & 1 & 2,7 \\
\hline Ninguno & 13 & 35 \\
\hline * En algunos casos se hicieron varios procedimientos en la misma paciente, \\
razón por la que la suma total de porcentajes es superior al $100 \%$.
\end{tabular}


muchos procedimientos que se realizaban con cirugía abierta tradicional, tales como: apendicectomía, fundo aplicación, herniorrafia, exploración de canal inguinal contralateral, linfadenectomía, orquidopexia, varicocele, esplenectomía, resección de vejiga, colecistectomía, escisión de divertículo de Meckel, gastrostomía, procedimientos anti reflujo, acalasia, estenosis pilórica, duplicación intestinal, mal rotación intestinal, síndrome heterotácico, intususcepción, anorectoplastia, adherencias intestinales, vólvulos gástrico, síndrome de arteria mesentérica superior y, trauma cerrado de abdomen.

Las primeras indicaciones en cirugía ginecológica fueron informadas por Cognat en 1973 en Francia, donde en un seguimiento a 3 años publicó 60 laparoscopias a niñas y adolescentes entre 6 y 19 años, la mayoría por problemas en el desarrollo sexual (46\%), el resto por amenorrea secundaria (16\%), defectos mullerianos (15\%), y dolor pélvico $(10 \%){ }^{7}$

La técnica quirúrgica de la laparoscopia tiene algunas diferencias en las adolescentes sobre todo en las delgadas y prepúberes y en las niñas. Se debe tener en cuenta que:

La vejiga de las niñas es un órgano intraperitoneal, por lo cual se recomienda para obviar posibles lesiones, localizar los tróqueres lateralmente, teniendo cuidado con la arteria epigástrica inferior y marcando el borde superior de la vejiga llenándola con agua a través del catéter foley.

La aorta: cuando la niña es delgada, la aorta está generalmente localizada justamente debajo de la piel debajo del ombligo y se puede lacerar accidentalmente simplemente haciendo la incisión umbilical con el bisturí. Para obviar esta complicación, el ombligo debe ser elevado por el operador con la mano, los dedos o con pinza de campo.

El útero: el uso del manipulador uterino no se debe usar en niñas prepúberes por el tamaño tan pequeño de vagina y útero. La posición supina es adecuada porque el acceso al útero y vagina no es usualmente necesario.

En 1992 Sanfilippo en Estados Unidos, señaló las principales indicaciones para laparoscopia en niñas y adolescentes: ${ }^{1}$
- Resección de embarazo ectópico

- Remoción de quiste ovárico

- Adhesiólisis

- Cirugía de procesos neoplásicos benignos

- Endometriosis

- Biopsia ovárica

Araya en $2002^{2}$ en un seguimiento de 5 años en Chile, encontró que se había practicado laparoscopia a 12 adolescentes en la mayoría por abdomen agudo $(33,3 \%)$, por dismenorrea severa en un $25 \%$ y por quiste pelviano en un $16,6 \%$.

Puelma en $2002^{3}$ en un seguimiento de 5 años en Chile, informó la realización de 9 cirugías laparoscópicas realizadas a niñas y adolescentes entre 6 a 16 años, la mayoría por quiste ovárico que no respondió a terapia médica con anticonceptivos (55\%), cuatro casos fueron indicados por quiste paraovárico, dolor pélvico, dismenorrea y malformación vaginal.

En Colombia, Ortiz en $2001^{4}$ en un seguimiento de 3 años en Profamilia Cali, informó la realización de 21 laparoscopias ginecológicas en adolescentes entre 15 a 19 años, la mayoría por dolor pélvico (67\%), el resto por infertilidad (19\%), amenorrea primaria y extracción de dispositivo intrauterino en cavidad abdominal. No hay informes de las otras clínicas que con frecuencia hacen cirugúa laparoscópica.

En nuestro estudio la frecuencia de este procedimiento en adolescentes en las 3 clínicas estudiadas es baja. La mayoría de las adolescentes llevadas a este procedimiento quirúrgico fueron adolescentes mayores que habían iniciado relaciones sexuales y que consultaban por dolor pélvico crónico o masas anexiales, encontrándose una proporción elevada de endometriosis lo que correlaciona con los estudios previos internacionales y locales. ${ }^{1-3}$

Llama la atención la presencia de infertilidad en este grupo de edad, lo que indica la edad muy temprana de inicio de relaciones y enfermedad pélvica inflamatoria con sus secuelas a nivel tubárico como el caso de la adolescente a la cual se le encontró hidrosálpinx y obstrucción tubárica, y el otro caso de embarazo ectópico que terminó en salpinguectomía. También llama la atención la exposición a complicaciones de anticoncepción 
con DIU y la presencia de varicocele no informado en otros estudios publicados.

\section{CONCLUSIONES}

El hallazgo más frecuente en las pacientes adolescentes sometidas a laparoscopia fue la endometriosis y patología ovárica benigna pero llama la atención el hallazgo de infertilidad, complicaciones de utilización de DIU y varicocele en mujeres tan jóvenes.

\section{Recomendaciones}

Estandarizar el registro de recolección de los datos de las historias clínicas prequirúrgicas intraoperatorias y posquirúrgicas para en el futuro tener unos parámetros idénticos para análisis y comparación en futuros estudios.

Hacer énfasis en la importancia que tiene la organización de los archivos de historias clínicas, para el conocimiento de la práctica de esta técnica quirúrgica.

Los autores del estudio no recibieron ningún tipo de financiación.

\section{AGRADECIMIENTOS}

Al Dr. Ney Guzmán, por la revisión del trabajo y asesoría en la fase de publicación.

\section{REFERENCIAS}

1. Sanfilippo JS. Operative Laparoscopy in the adolescent. Obstet Gynecol Clin North Am 1992;19:163-76.

2. Araya M, Díaz H, Fuentes L. Cirugía laparoscópica ginecológica en adolescentes. Revista de la Sociedad Chilena de Obstetricia y Ginecología Infantil y de la Adolescencia SOGIA 2002;9:27.

3. Puelma P, Oyarzun P, Contador M. Experiencia Laparoscópica en pacientes ginecológicas de la Unidad de Ginecología del Hospital de niñas y niños Roberto del Rió, durante el año 2000. Revista de la Sociedad Chilena de Obstetricia y Ginecología Infantil y de la Adolescencia SOGIA 2002;9:28.

4. Ortiz CA, Viafara G. Laparoscopia ginecológica en mujeres adolescentes: experiencia institucional durante 4 años (1998-201) en la Clínica de Profamilia de Cali, Colombia. Revista de la Sociedad Chilena de Obstetricia y Ginecología Infantil y de la Adolescencia SOGIA 2002;9:26.

5. Cognat M, Rosenberg D, David L, Papathanassiou Z. Laparoscopy in infants and adolescents. Obstet Gynecol 1973;42:515-21.

6. Sharp HT.Laparoscopy in children. Clin Obstet Gynecol, 1997;40:210-8.

7. Zitsman JL. Current concepts in minimal access surgery for children. Pediatrics 2003;111:1239-52. 